\title{
Mixed Nonseminomatous Germ Cell Tumor with Rhabdomyosarcomatous Malignant Transformation in a Pediatric Patient
}

\begin{abstract}
Testicular germ cell tumors (GCTs) are the most common malignancy in males aged 15-34. The transformation of GCTs into secondary somatic-type malignancies is rare, and the lack of clear treatment guidelines presents a clinical challenge for treating physicians, especially when chemosensitivities do not overlap. We present the case of a 15-year-old-male with a mixed nonseminomatous malignant GCT with an embryonal rhabdomyosarcoma component, which made up $60 \%$ of the primary tumor. He was successfully treated with surgical resection and chemotherapy. We highlight our experience in diagnosing and treating this tumor, and through literature review, suggest treatment guidelines for treating a pediatric patient with similar tumor presentation.
\end{abstract}

Keywords: Pediatrics, rhabdomyosarcoma, secondary somatic-type malignancy, testicular germ cell tumor

\section{Introduction}

Testicular germ cell tumors (GCT) are the most common malignancy in males aged 15-34. ${ }^{[1]}$ The transformation of testicular GCTs into secondary somatic-type malignancies is rare. We present an unusual case of a mixed nonseminomatous malignant GCT with an embryonal rhabdomyosarcoma (RMS) component in a 15-year-old male. We highlight our experience in diagnosing and treating such a tumor at a pediatric institution, utilizing Children's Oncology Group (COG) protocol.

\section{Methods and Materials}

The patient's medical records were accessed and reviewed at Cook Children's Medical Center in Fort Worth, Texas.

\section{Case Report}

Two months following surgical repair of bilateral varicocele, a 15-year-old male with medical history remarkable for Osgood-Schlatter disease complained of a painless, hard mass involving his right testicle. A right radical orchiectomy was performed, and postsurgical resection was achieved with negative margins. Histopathologic examination of the mass sized $3.3 \mathrm{~cm} \times 2.8 \mathrm{~cm} \times 2.3 \mathrm{~cm}$, showed

This is an open access article distributed under the terms of the Creative Commons Attribution-NonCommercial-ShareAlike 3.0 License, which allows others to remix, tweak, and build upon the work non-commercially, as long as the author is credited and the new creations are licensed under the identical terms.

For reprints contact: reprints@medknow.com a mixed malignant GCT with a secondary somatic-type malignant component. There were large areas of a primitive small cell malignancy with pleomorphic cells containing variable amount of eosinophilic cytoplasm [Figure 1]. A few large "strap" cells resembling fetal muscle were seen. Immunostaining of the tumor confirmed these sarcomatous areas were highlighted with the myogenic markers desmin and myogenin. The tumor did not express the GCT markers OCT3/4, placental alkaline phosphatase, or alpha-fetoprotein (AFP). The tumor constituted the following: teratoma (20\%), embryonal RMS (60\%), embryonal carcinoma (15\%), and yolk sac tumor $(<5 \%)$. The tumor was limited to the testis and epididymis, extending to the tunica albuginea but not the tunica vaginalis. There was no evidence of vascular or lymphatic invasion. In addition, ipsilateral retroperitoneal lymph node dissection, computed tomography (CT) scans of the chest, abdomen, pelvis, and metastases.

The mixed GCT component was deemed Stage I because of complete resection and lack of metastasis; the RMS component was also deemed Stage I due to location in nonbladder/prostate genitourinary tract and Group I due to complete surgical resection

\footnotetext{
How to cite this article: Pham RL, Ray $A$, Margraf LR. Mixed nonseminomatous germ cell tumor with rhabdomyosarcomatous malignant transformation in a pediatric patient. Indian J Med Paediatr Oncol 2018;39:250-3.
} bone scan showed no evidence of distant

\author{
Robin L Pham, \\ Anish Ray ${ }^{1}$, \\ Linda R Margraf ${ }^{1}$ \\ University of North Texas \\ Health Science Center, \\ ${ }^{1}$ Cook Children's Medical \\ Center, Fort Worth, TX, USA
}

Address for correspondence: Miss. Robin L. Pham, 3500 Camp Bowie Boulevard Fort Worth, Texas, 76107, USA. E-mail: rlp0184@my.unthsc.edu

Access this article online Website: www.ijmpo.org DOI: 10.4103/ijmpo.ijmpo_207_17 Quick Response Code:

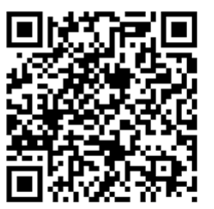




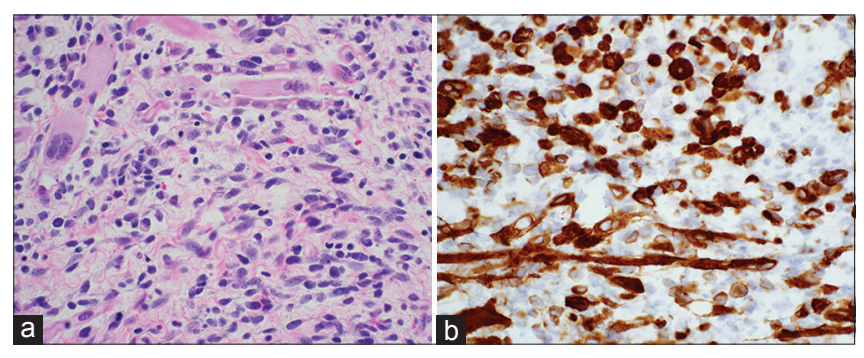

Figure 1: (a) Photomicrograph of the tumor shows areas of primitive small cells with occasional enlarged "strap" cells showing skeletal muscle differentiation. Mitoses are abundant (hematoxylin and eosin, $\times 400$ ). (b) Immunohistochemical staining for desmin confirms muscle differentiation in the areas small cell malignancy $(\times 400)$

of the mass. Initial laboratory values showed that AFP and human chorionic gonadotropin (hCG) were not elevated. We characterized this testicular tumor as low risk.

We then targeted the RMS component with chemotherapy, utilizing COG protocol ARST0331: 4 cycles of vincristine, dactinomycin, and cyclophosphamide followed by 4 cycles of vincristine and dactinomycin. Following 24 weeks of therapy, the patient's CT scans of the chest, abdomen, and pelvis were negative, and AFP and hCG laboratory values remained unremarkable. Approximately 34 months posttherapy, the patient remains in good health and has shown no evidence of tumor recurrence. Of note, the patient has sustained recurrence of bilateral varicocele.

\section{Discussion and Conclusion}

Childhood extracranial GCTs, arising from primordial germ cells, are broadly classified into teratomas, malignant GCTs, and mixed malignant GCTs. Malignant GCTs are subdivided into seminomatous and nonseminomatous GCTs. Seminomatous GCTs are further classified into seminoma, dysgerminoma, and germinoma; nonseminomatous GCTs are divided into yolk sac tumor, choriocarcinoma, embryonal carcinoma, and gonadoblastoma. ${ }^{[2]}$

The development of a secondary somatic-type malignant component from a primary GCT is a documented phenomenon; however, those arising from testicular masses are rare. ${ }^{[3]}$ Possible secondary somatic-type malignancies include adenocarcinoma, primitive neuroectodermal tumor, RMS, and leukemia. ${ }^{[4]}$ It is presumed that the RMS arises from the teratoma component of the mixed germ cell neoplasia. Teratomas in postpubertal males are malignant and often contain highly atypical areas resembling sarcomas, but these are generally intermixed with other more mature teratomatous elements. To be accurately classified as a "teratoma with somatic-type malignancy" requires overgrowth of the sarcoma, or less commonly carcinoma, to occupy at least a low power $(\times 4)$ field.${ }^{[5]}$ The growth may be nodular or infiltrative. ${ }^{[3]}$ In our case, the primitive myogenic sarcoma comprised more than half of the neoplasm.

Primitive malignant sarcomas are seen in rare cases of spermatocytic seminoma, including a few where the sarcoma is a RMS; however, these tumors generally arise in older adult men and have a seminoma component, which was not seen in our case. ${ }^{[5]}$ Yolk sac tumors may also occasionally harbor a component of malignant sarcoma. On occasion, metastatic disease will harbor sarcomatous elements, not seen in the primary neoplasm. ${ }^{[3]}$

Currently, there are several reported cases that demonstrate the challenge of treating patients with testicular tumors with associated rhabdomyosarcomatous components. The major challenge in treating these tumors is that the chemosensitivities of GCT and RMS do not overlap; GCT is sensitive to cisplatin while RMS is not. ${ }^{[3]}$ One of the earliest reports of sarcomatous differentiation from a testicular GCT shows that following eradication of the primary germ cell component by cisplatin therapy, the sarcomatous component, which was a minor component in the initial specimen, became the predominant species. ${ }^{[6]}$ Stamatiou et al. also describe a similar case with the treatment of the GCT with cisplatin therapy. The patient, in this case, died after metastasis-related complications. ${ }^{[7]}$ These two aforementioned cases highlight that although GCT may be the primary tumor, failure to consider the sarcomatous component in therapy may be deadly to the patient. Remaining reports highlight various methods of therapy, for example: one targeting the GCT component, one targeting the rhabdomyosarcomatous component, and one utilizing surgical resection without chemotherapy. ${ }^{[3,6,8]}$ These cases demonstrate the lack of clear treatment guidelines.

Our greatest challenge in prescribing treatment for this tumor was understanding how to target the disparate components. Current literature suggests that active surveillance strategies are the recommended treatment for Stage I testicular GCT due to their effectiveness and minimization of adverse effects from chemotherapy and radiation. ${ }^{[9]}$ Because this patient's primary testicular GCT was Stage I, we chose to follow the strategy of COG study AGCT0132, which describes that surgery and surveillance are sufficient in managing patients with low-risk non-seminoma tumors. ${ }^{[1]}$ Furthermore, we targeted the RMS component with chemotherapy as indicated in the current literature. According to COG protocol ARST0331, in cases of low-risk RMSs, a 24-week therapy schedule is recommended with 4 cycles of vincristine, dactinomycin, and cyclophosphamide followed by 4 cycles of vincristine and dactinomycin. ${ }^{[10]}$ We followed this protocol, and the patient finished treatment without any complication.

Regardless, we recognize that such cases remain tremendously challenging, given the lack of consensus in treating two malignancies whose chemosensitivities do not overlap. There is little debate over the fact that successful surgical resection aimed toward securing negative margin remains key inadequate treatment of those with localized disease. ${ }^{[1]]}$ With regard to choice of chemotherapy postoperatively, there is some suggestion that malignant transformation of GCT responds poorly to cisplatin-based 


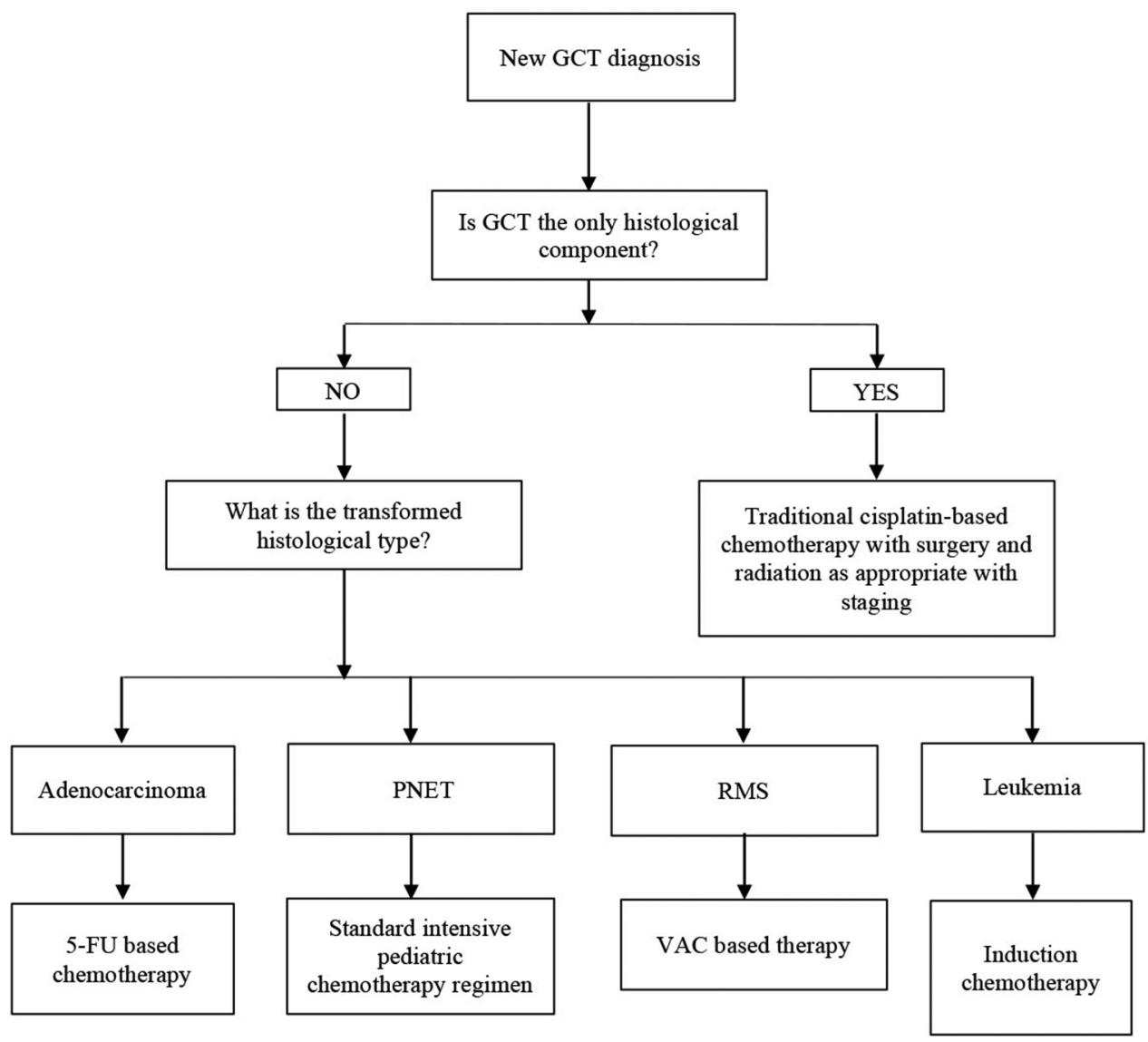

Figure 2: Proposed treatment guideline for new germ cell tumor diagnosis in pediatric patient. GCT - Germ cell tumor; PNET - Primitive neuroectodermal tumor; RMS - Rhabdomyosarcoma; 5-FU - 5-fluorouracil; VAC - Vincristine, dactinomycin, cyclophosphamide

therapy ${ }^{[4]}$ In fact, Donadio et al., based on the review of 12 patients, suggest that choice of chemotherapy should be guided by transformed histology unless there is residual GCT in conjunction with the malignantly transformed element. ${ }^{[4]}$ In another paper, Korfel et al. describe treating a patient with a testicular GCT and widely metastatic RMS with chemotherapy consisting of epirubicin, ifosfamide, etoposide, and cisplatin followed by high-dose chemotherapy and stem-cell rescue, resulting in complete response lasting several years. ${ }^{[12]}$ Based on literature review, in treating a pediatric patient, we suggest that choice of chemotherapy agents should be influenced by the transformed histological element as the transformed element may not be responsive to cisplatin-based therapy [Figure 2].

Our report has limited value given singular patient; however, reporting on large series of such patients will be challenging given the rarity. We suggest developing a multi-institutional trial to include both adolescents and adults whose outcomes may be followed closely to optimize treatment for future patients.

\section{Declaration of patient consent}

The authors certify that they have obtained all appropriate patient consent forms. In the form the patient(s) has/have given his/her/their consent for his/her/their images and other clinical information to be reported in the journal. The patients understand that their names and initials will not be published and due efforts will be made to conceal their identity, but anonymity cannot be guaranteed.

\section{Financial support and sponsorship}

Nil.

\section{Conflicts of interest}

There are no conflicts of interest.

\section{References}

1. Hayes-Lattin B, Nichols CR. Testicular cancer: A prototypic tumor of young adults. Semin Oncol 2009;36:432-8.

2. Mikuz G. Germ cell and sex cord-stromal tumors of the testis: WHO classification 2016. Pathologe 2017;38:209-20.

3. Guo CC, Punar M, Contreras AL, Tu SM, Pisters L, Tamboli P, et al. Testicular germ cell tumors with sarcomatous components: An analysis of 33 cases. Am J Surg Pathol 2009;33:1173-8.

4. Donadio AC, Motzer RJ, Bajorin DF, Kantoff PW, Sheinfeld J, Houldsworth $\mathrm{J}$, et al. Chemotherapy for teratoma with malignant transformation. J Clin Oncol 2003;21:4285-91.

5. Ulbright TM, Young RH. Tumors of the Testis and Adjacent Structures, in AFIP Atlas of Tumor Pathology. Ser. 4. Silver Spring, Maryland: American Registry of Pathology Press; 2013.

6. Ulbright TM, Loehrer PJ, Roth LM, Einhorn LH, Williams SD, Clark SA, et al. The development of non-germ cell malignancies 
within germ cell tumors. A clinicopathologic study of 11 cases. Cancer 1984;54:1824-33.

7. Stamatiou K, Papadopoulos P, Perlepes G, Galariotis N, Olympitis M, Moschouris H, et al. Mixed germ cell tumor of the testicle with ravdomuosarcomatous component: A case report. Cases J 2009;2:9299.

8. Lovrić E, Hizak DB, Balja MP, Lenicek T, Kruslin B. An unusual mixed germ cell tumor of the testis consisting of rhabdomyosarcoma, mature teratoma and yolk sac tumor. Asian J Androl 2010;12:451-2.

9. Nichols CR, Roth B, Albers P, Einhorn LH, Foster R, Daneshmand $\mathrm{S}$, et al. Active surveillance is the preferred approach to clinical stage I testicular cancer. J Clin Oncol 2013;31:3490-3.
10. Malempati S, Hawkins DS. Rhabdomyosarcoma: Review of the children's oncology group (COG) soft-tissue sarcoma committee experience and rationale for current COG studies. Pediatr Blood Cancer 2012;59:5-10.

11. Faure Conter C, Fresneau B, Thebaud E, Bertrand A, Dijoud F, Rome A, et al. Two tumors in 1: What should be the therapeutic target? Pediatric germ cell tumor with somatic malignant transformation. J Pediatr Hematol Oncol 2017;39:388-94.

12. Korfel A, Fischer L, Foss HD, Koch HC, Thiel E. Testicular germ cell tumor with rhabdomyosarcoma successfully treated by disease-adapted chemotherapy including high-dose chemotherapy: Case report and review of the literature. Bone Marrow Transplant 2001;28:787-9. 